\title{
Statistical Modelling of Mechanical Tensile Properties of Steels by Using Neural Networks and Multivariate Data Analysis
}

\section{Christian DUMORTIER and Philippe LEHERT ${ }^{1)}$}

Metallurgy Department, Polytechnic Faculty of Mons, 56 rue de l'Epargne, 7000 Mons Belgium.

1) Data Processing Department, Catholic University of Mons, 151 Chaussée de Binche,7000 Mons Belgium.

(Received on February 18; accepted in final form on June 14, 1999)

Both Neural networks NN and Multivariate Data Analysis MDA techniques were used to determine mechanical tensile properties of steels. $\mathrm{NN}$ apparently proved better in dealing with non-linearity effects and complex interactions between the predictors. However, MDA was found essential in improving the quality of the prediction and providing interpretation to the results from NN modelling.

KEY WORDS: artificial neural network; mechanical properties; microalloyed steel; multiple regression; multivariate data analysis; principal components analysis.

\section{Introduction}

Neural Networks (NN) modelling becomes a widely used technique in Material science, for various tasks such as estimation of the values of different properties, multinomial prediction from the values of a set of parameters or classification on a collection of objects or events. Due to its popularity, NN is now available in most of commercial Statistical and Multivariate Data Analysis (MDA) packages.

More classical statistical MDA techniques aim at the same purposes, and in particular Multiple Regression for estimation and prediction, Discriminant and Cluster Analyses for classification.

In this paper, we compare the relative ability of multiple regression and $\mathrm{NN}$ in the particular problem of estimation and prediction of mechanical tensile properties of steels.

It is known that several interacting and complex mechanisms of action ${ }^{1,2)}$ contribute to explain steel mechanical properties: contributions relative to solid solution hardening, strengthening by precipitation particles and microstructure, grain size effect and dislocations.

The induced multifactorial data structure is probably the major obstacle in building a theoretical predictive model under general conditions. This complexity also justifies the use a statistical approach based on observed samples and often approached by general least square adjustments, in particular multiple regression models. $^{1,3,4)}$

Compared with Multiple Regression, NN was observed more versatile in dealing with non-linearity effects and interactions between predictors. However, it does not provide in any way an explanatory formal model between the dependent used variable and the predictors.
Another major drawback is the possibility of bad predictions during the validation step due to the multiplicity of parameters of the model. This necessitates caution during the learning phase.

In this paper, we emphasise the complementary interest of MDA in improving the quality of the prediction and providing an interpretation to the $\mathrm{NN}$ results, by using as illustration a recent practical study case from Cockerill-Sambre Steel plant. ${ }^{5)}$

\section{Methods}

\subsection{Backpropagation Neural Networks ${ }^{6-10)}$}

Neural nets are interconnected agglomerates of simple computing units call neurones or nodes. A symbolic representation of a direct network is given in Fig. 1. The input of any neurone $i$ is given by:

$$
e_{i}(t)=\sum w_{i j}(t) a_{j}(t)+\theta_{i}
$$

$a_{j}(t)$ is the activation state of the $j$ interconnected nodes, $w_{i j}$ is the input weight from input node $i$ to node $j$ and $\theta_{i}$ an offset or a bias term. The most used activation sigmoïd function is:

$$
a_{i}(t+1)=\frac{1}{1+e^{-e i(t)}}
$$

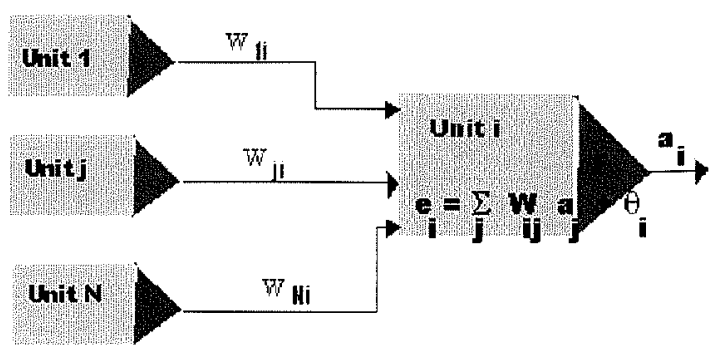

Fig. 1. Symbolic representation of a direct NN network. 


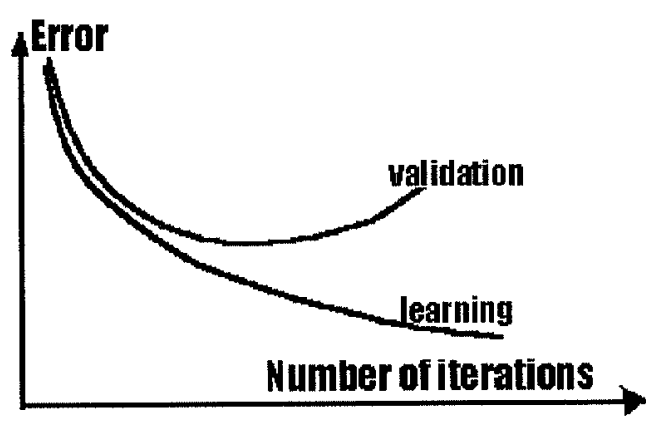

Fig. 2. Behaviour of $E(W)$ error computed on training and validation datasets.

In this work, a feed-forward network performing the classical back-propagation training algorithm with the generalised delta rule with momentum has been used. $\mathrm{NN}$ acquire their knowledge during a learning phase. During this step, NN compare the target values $t_{j}$ with the desired output values $y_{j}$ and the weight values $w_{i j}$ (initially fixed by a random procedure) are modified in order to achieve the final estimation goal. For a set $W$ of given weight values and for $n$ patterns, the error between the $M$ target values $t_{j}$ and the estimated values $y_{j}$ is measured by the following expression:

$$
E(W)=\frac{1}{M \cdot n} \sum_{i=1}^{n} \sum_{j=1}^{M}\left(y_{i j}-t_{i j}\right)^{2}
$$

In the learning step, the $w_{i j}$ must be considered as parameters, whose values are adjusted repeatedly by the back-propagation algorithm until the root mean square error $E(W)$ fall under a predetermined value.

In choosing a very small value, the error function always exceeds this level and the training procedure resumes until it matches the selected number of iterations. Moreover, the best results were obtained for separated trainings one for each mechanical property considered $(M=1)$.

The action of the network is determined by its architecture (input size, hidden and output nodes) and the values of the weights. The input and output nodes depend on the application while the number of hidden nodes is a variable adjusted by the user. Setting the number and size of hidden layers is not an easy task. If too few hidden units are used, the network may fail to learn properly. Conversely, if too many hidden units are used, the data may be overfitted and this consequently reduces the network's ability to generalise for new samples. ${ }^{6)}$ For a fixed and sufficient number of iterations, the error function generally decreases in the learning phase when the weight number related to the number of hidden nodes increases. Similarly, for a fixed number of hidden units, the error function decreases during the learning step when the number of iterations raises.

In this study, to deal with the over-fitting problem, the ratio of number of patterns of the learning data set to the number of hidden units has been chosen larger than 20 and the training phase has been stopped when the error function (1) calculated on a separate (and first validation) database begins to increase (Fig. 2).

Finally, a last check was conducted on an independent

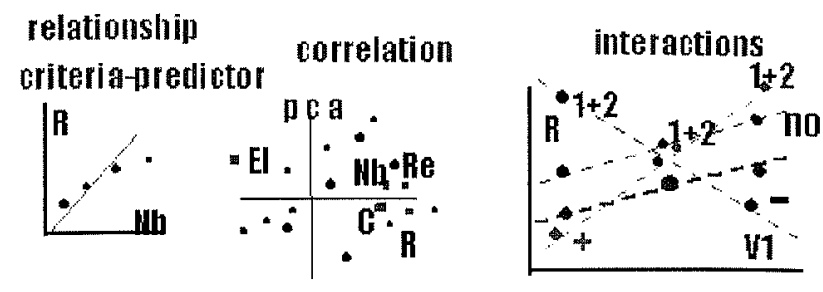

\section{new predictors}

Ar3 $=910-310 \%$ in $-80 \%$ liln

\section{categorical variables}

spec. A, B, G $\rightarrow$ 3 var $0 / 1$
Fig. 3. A priori knowledge for a regression model.

(or second) validation database.

\subsection{Multivariate Data Analysis}

Regression is perhaps the most widely used technique for assessing linear models. The multiple regression model $y=b_{1} x_{1}+\cdots+b_{p} x_{p}+a$ was estimated by means of a forward technique of selection of variables.

However, using Regression requires a priori knowledge which involves data pre-processing including different steps schematised at Fig. 3:

- introduction of dummy variables in order to take into account the effect of categorised variables

- detection of non-linear effects between dependent and independent variables and creation of appropriated variables when such effects are observed

- detection of interactions between independent variables and formulation of their mathematical expression.

In this application, significant non-linearity and interaction effects were not detected so only dummy variables were introduced into the regression models.

When using $\mathrm{NN}$, all the preliminary treatments schematised at Fig. 3 are largely taken into account by the multilayers structure.

However, transformations must be realised on variables in order to convert them on a $0-1$ range.

Another crucial problem on which final model estimation is sensitive is data reliability or abnormal/extreme values existence. Figure 4 exhibits one particular case of data reliability problem. Often, the sensitivity to extremes is such that outliers detection must take place on both learning and validation data sets. Classically, the detection of outliers is first carried out for each variable by reference to physical considerations or by use of statistical tools like histograms or boxplots. ${ }^{11)}$ But, this approach must be completed in $p$-space ( $p$ being the number of inputs connected to the first layer) to detect cases distant from the centroïd or belonging to unexplored space regions. In this purpose, the multivariate Mahalanobis distance ${ }^{12)}$ detects outliers as multivariate measurements outside $(1-\alpha) \cdot 100$ Confidence Ellipsoids.

Principal Components Analysis ${ }^{13)}$ (PCA), whose first purpose is to give a representation of all variables in a (factorial) space of limited dimensionality $k(k \ll p)$ is also useful to detect the abnormal cases. ${ }^{14)}$ In this study, PCA has also been used to represent the cases of the validation databases in the factorial space construct- 

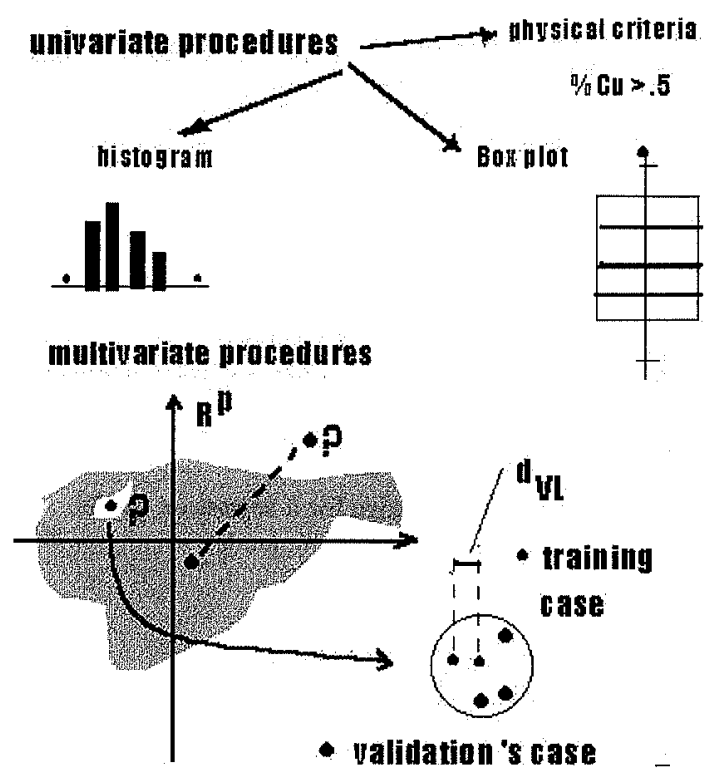

Fig. 4. Statistical analysis focused on data reliability.

ed from the training data set

\section{Material}

The basic material data is constituted by 12400 carbon and HLSA steel coils suitable for construction and car production and collected over 3 years (1995-1997) at Cockerill-Sambre Carlam Steel Plant on the hot strip mill (Carlam). Material data include the following characteristics (symbol of variables within brackets):

- mechanical tensile properties (Yield strength [Re], Ultimate Strength $[R]$, Elongation $[E l]) ;$

- mechanical tests (thickness [THb], type of normalised specimens [TS_T] (with 2 categories ISO 25/125 and ISO 20/80) and position in the coil of the test specimen [TS_P] (with 3 categories top, bottom, middle); - plate dimensions (thickness [THI], width [W] );

- steel chemical analysis $([\mathrm{C}],[\mathrm{Mn}],[\mathrm{P}],[\mathrm{S}],[\mathrm{Si}],[\mathrm{Al}]$, $[\mathrm{V}],[\mathrm{Nb}],[\mathrm{Cu}],[\mathrm{Cr}],[\mathrm{Ni}],[\mathrm{N}],[\mathrm{Ti}],[\mathrm{Sn}],[\mathrm{B}]$ contents);

- rolling process (reheating $\left[\mathrm{T}^{\circ} \mathrm{F}\right]$, roughing $\left[\mathrm{T}^{\circ} \mathrm{S}\right]$, finishing $\left[\mathrm{T}^{\circ} \mathrm{R}\right]$ and coiling $\left[\mathrm{T}^{\circ} \mathrm{C}\right]$ temperatures).

Among the available variables, only 20 have been selected for the final statistical modelling (17 predictors and 3 criteria). The reason is that preliminary regression and neural networks tests have shown that the remaining ones had a very limited influence on the mechanical properties. Among other deleted variables, we mention in particular those associated with the residual elements $(\mathrm{P}, \mathrm{S} \cdots)$.

\section{Results}

All the statistical treatments including data preparation and the neural network calculations have been carried out with Fortran and $\mathrm{C}$ codes running on PCPentium and DEC 3000 station.

About 200 cases were a priori excluded to abnormal or missing values so finally 12197 cases were found eligible for the analysis. In a first separation, those cases
THI C MnSi Nb V Ti $B$ AI $N$ Cu TS TEMP.

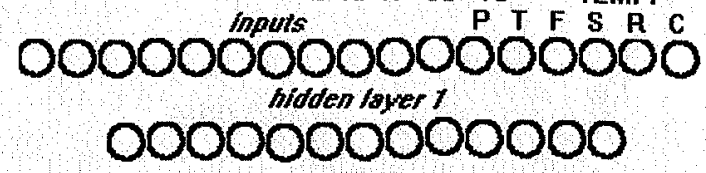
bidoten lower 2 ? 0000000 ootputs Doo

A Re El

Fig. 5. Representation of the two hidden layers $\mathrm{NN}$ used for the modeling.

Table 1. Comparison of results obtained by neural networks and multiple linear regression.

\begin{tabular}{|lcccc|}
\hline NEURAL NETWORK & itarations & 2000 & 2500 & 8000 \\
Learning dataset & $\mathrm{n}=10000$ & $\sigma_{\mathrm{Re}} 17.9 \mathrm{~N} / \mathrm{mm}^{2}$ & $\sigma_{\mathrm{El}} 3.05 \%$ & $\sigma_{\mathrm{R}} 12.6 \mathrm{~N} / \mathrm{mm}^{2}$ \\
$1^{\text {st }}$ valid. dataset & $\mathrm{n}=697$ & $\sigma_{\mathrm{Re}} 20.3 \mathrm{~N} / \mathrm{mm}^{2}$ & $\sigma_{\mathrm{El}} 3.05 \%$ & $\sigma_{\mathrm{R}} 14.5 \mathrm{~N} / \mathrm{mm}^{2}$ \\
$2^{\text {nd }}$ valid. dataset & $\mathrm{n}=1500$ & $\sigma_{\mathrm{Re}} 22.4 \mathrm{~N} / \mathrm{mm}^{2}$ & $\sigma_{\mathrm{El}} 3.38 \%$ & $\sigma_{\mathrm{R}} 15.4 \mathrm{~N} / \mathrm{mm}^{2}$ \\
MULTIPLE LINEAR REGRESSION & & & \\
Learning dataset & $\mathrm{n}=10000$ & $\sigma_{\mathrm{Re}} 22.5 \mathrm{~N} / \mathrm{mm}^{2}$ & $\sigma_{\mathrm{El}} 3.52 \%$ & $\sigma_{\mathrm{R}} 16.9 \mathrm{~N} / \mathrm{mm}^{2}$ \\
$1^{\text {st }}$ valid. dataset & $\mathrm{n}=697$ & $\sigma_{\mathrm{Re}} 23.2 \mathrm{~N} / \mathrm{mm}^{2}$ & $\sigma_{\mathrm{El}} 3.35 \%$ & $\sigma_{\mathrm{R}} 17.1 \mathrm{~N} / \mathrm{mm}^{2}$ \\
$2^{\text {nd }}$ valid. dataset & $\mathrm{n}=1500$ & $\sigma_{\mathrm{Re}} 26.0 \mathrm{~N} / \mathrm{mm}^{2}$ & $\sigma_{\mathrm{El}} 3.50 \%$ & $\sigma_{\mathrm{R}} 18.0 \mathrm{~N} / \mathrm{mm}^{2}$ \\
\hline
\end{tabular}

were arbitrarily classified in two groups of 10697 and 1500 items corresponding to coils produced before and after June 1997. The first group is used for the training phase and the second constitutes the independent validation data set.

By using a random allocation, a second separation was considered, the 10697 cases being distributed in two groups, the larger $(10000)$ reserved for the neural network training step while the less important (697) aimed to give a criterion to stop the learning phase (cf. Fig. 2) as explained before.

\subsection{NN Results}

The NN architecture selected for the mechanical properties modelling is depicted in a schematic way at Fig. 5. To characterise the NN estimation of the three mechanical criteria the following parameters have been computed: the error function (1), the coefficient correlation between calculated and measured values $\left(r_{\mathrm{CM}}\right)$ and their standard error of estimate $\sigma$. The final results of the training and validation results are shown in Table 1. It can be shown that standard error of estimate are greater for the two validation data sets but that the results remain good and significantly better than those obtained by application of a multiple linear regression model including 3 dummy variables.

\subsection{MDA Results}

The application of multiple regression on ultimate strength exhibits as predictors, in decreasing order: $\mathrm{Nb}(+$ effect), $\mathrm{C}(+), \mathrm{Mn}(+), \mathrm{N}(+)$, Coiling_- $\mathrm{T}^{\circ}(-), \mathrm{Ti}(+)$, $\mathrm{B}(-), \mathrm{V}(+)$ and $\mathrm{Si}(+)$. For a visualisation of the 


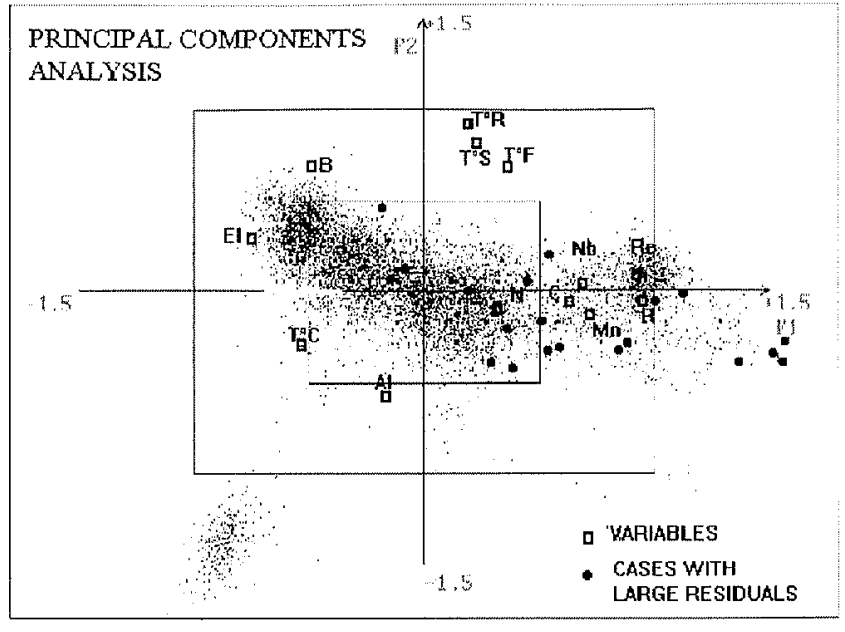

Fig. 6. PCA factor plot $F_{1}-F_{2}$

relationships between all input quantitative variables, PCA was used. On the main factor Plot $F_{1}-F_{2}$ (Fig. 6), the position of two variables $V_{1}$ and $V_{2}$ is such that their correlation is measured by the cosine of the angle $O V_{1} V_{2}$ ( $\mathrm{O}$ is origin). Close variables as $\mathrm{Re}$ and $\mathrm{R}$ are strongly correlated $(r=0.97)$, and also very close to $\mathrm{Nb}$, the three variables contributing to the first component $F_{1}$. Factor plot $F_{1}-F_{2}$ also reveals that elongation has an important and negative loading on the first axis, which is due to the deleterious effect on ductility of an increase on tensile strengthening by precipitation.

PCA also enables a visualisation of the cases of the sample by projecting them on the components (factor scores). As suggested by Fig 6, the training database is not homogeneous since large clusters can be detected in the multidimensional space. For example coils for pipes are gathered in a cluster situated on the right side of the first principal axis and are characterised by high values of $\mathrm{Re}, \mathrm{R}, \mathrm{C}, \mathrm{Nb}$ and low values of coiling temperature $\left(T^{\circ} \mathrm{C}\right)$. An other cluster whose cases have negative scores on the two axis correspond to low carbon stabilised steels whereas steels for deep drawability applications (with boron addition and $\mathrm{T}^{\circ} \mathrm{C}$ values ranging between 650 and $750^{\circ} \mathrm{C}$ ) belong to a cluster situated on the left side on the first axis.

\section{Discussion}

The results obtained by $\mathrm{NN}$ are less suggestive for several reasons. First the number of parameters of the NN model (weight values) is considerably higher than in multiple regression $13 \cdot(17+1)$ for the first layer, $7 \cdot(13+1)$, for the second one and $(7+1)$ for the output layer. On the other hand, those values change with the number of iterations during the training phase as it can be observed in Table 2 where connection weights between 3 nodes of layer 1 to input variable $\mathrm{Nb}$ are given.

In this example, the contribution of nodes 10 and 12 is essential whereas node 2 is less far important. Table 2 also suggests that $\mathrm{Nb}$ connection weights of nodes 10 and 12 evolve in the same way and seem rather substitutable. Finally, for weights of layers 2 and 3, the interpretation is still more difficult because the cor-
Table 2. Evolution of the connection weights between $\mathrm{Nb}$ and nodes 2, 10 and 12 of hidden layer 1 during the training.

\begin{tabular}{|l|c|c|c|c|c|c|}
\hline \multicolumn{1}{|c|}{ Iterations } & 500 & 1500 & 2500 & 5000 & 8000 & 10000 \\
\hline$\sigma \mathrm{N} / \mathrm{mm}^{2}$ & 14.4 & 13.4 & 13.2 & 12.9 & 12.6 & 12.4 \\
Weights $\rightarrow$ & & & & & & \\
Node 2 & -0.129 & -0.247 & -0.148 & -0.23 & 0.17 & 0.175 \\
Node 10 & 0.14 & 0.113 & 0.343 & 1.186 & 2.702 & 3.389 \\
Node 12 & -1.11 & -1.189 & -1.335 & -0.665 & 0.193 & 0.495 \\
\hline
\end{tabular}

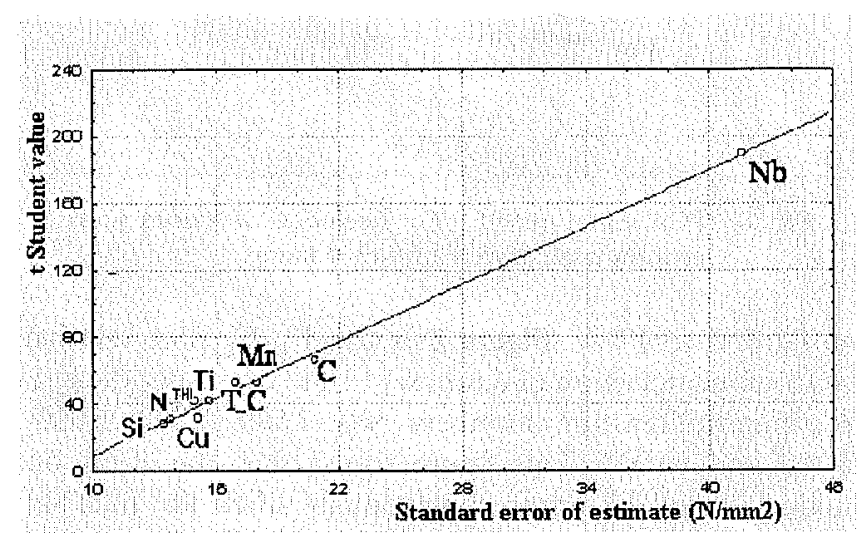

Fig. 7. Prediction of ultimate strength.

responding values are not direct functions of input variables but depend themselves on the values of the connection weights of the previous layers (1 and 2).

\subsection{Interpreting NN Results}

To evaluate the influence of an input variable on the estimated values given by the NN model, the simplest way is maybe to fix the value of this variable to a constant level for all cases of the data set (training or validation).

In this condition, the stronger the effect of the selected input variable on the mechanical property considered, the higher the standard error of estimate $\sigma$ obtained by NN modelling. As an example, for the training data set $(n=10000)$ and for the ultimate strength $\sigma$ increases from a reference value of $12.6 \mathrm{~N} / \mathrm{mm}^{2}$ (value given in table 1 for an iteration number equal to 8000 ) to 41.6 $\mathrm{N} / \mathrm{mm}^{2}$ when niobium content is replaced by a constant.

Niobium is by far the most influential strength factor since $\sigma$ values for the most other significant variables after $\mathrm{Nb}$ are only $20.8 \mathrm{~N} / \mathrm{mm}^{2}$ for $\mathrm{C}, 17.9 \mathrm{~N} / \mathrm{mm}^{2}$ for $\mathrm{Mn}$ and $16.9 \mathrm{~N} / \mathrm{mm}^{2}$ for the coiling temperature. Figure 7 shows that computed signification $t$ Student values of the regression coefficients used to are in accordance with values of standard error of estimate by $\mathrm{NN}$ of ultimate strength.

The following paragraph relates to the interpretation of NN results in term of explanation of the weight values and particularly their evolution when the number of iterations increases.

A multivariate approach of the problem is to consider that a node of layer 1 can be represented by a point in a 18-space (17 input variables and 1 offset). With this concept, the values of the 13 nodes of layer 1 corresponding to a given number of iterations must constitute a table of data of 13 rows which can be again 


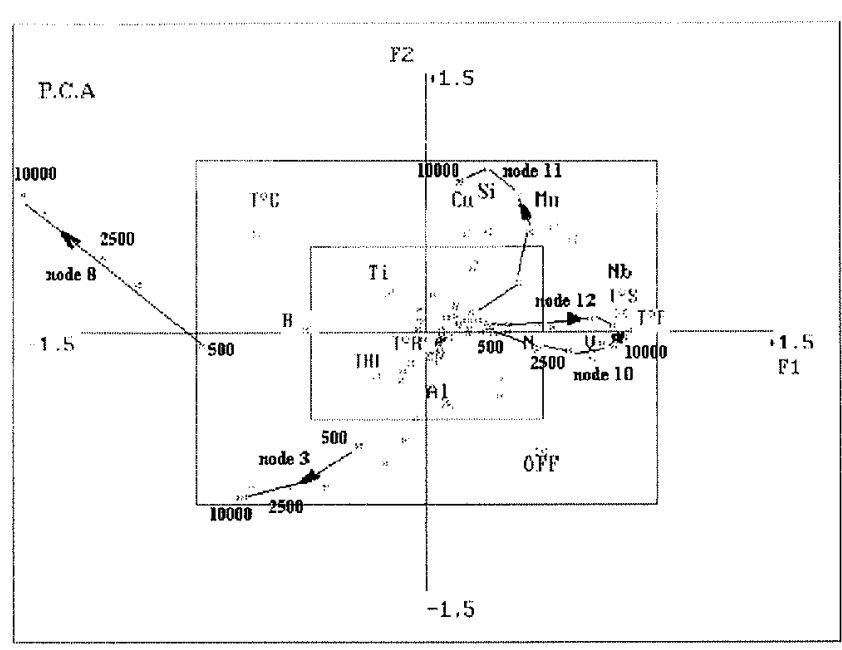

Fig. 8. PCA factor plot $F_{1}-F_{2}$. Evolution of hidden units contributions (layer 1 ) during training.

submitted to PCA. Figure 8 is the $F_{1}-F_{2}$ plot relative to the concatenation of 6 tables $[13,18]$ corresponding to 6 different iteration numbers 500, $1500,2500,5000$, 8000 and 10000 . This figure shows that the effect on nodes 10 and 12 raises continuously when the number of iterations increases from 500 to 10000 . Those two nodes are characterised by increasing values of $\mathrm{Nb}, \mathrm{N}$, roughing $\left(\mathrm{T}^{\circ} \mathrm{S}\right)$ and reheating $\left(\mathrm{T}^{\circ} \mathrm{F}\right)$ temperatures. The difference of behaviour between node 10 and 12 is explained on principal axis 5 which is characterised by N and Ti variables, the connection weights "node 12-Ti" being more negative than those "node 10-Ti" and the connection values "node $12-N$ " being more positive than for node 10.

Figure 8 also suggests that node 8 plays a major role for deep drawability steels since it corresponds to high values of $\mathrm{B}$ and coiling temperature $\left(\mathrm{T}^{\circ} \mathrm{C}\right)$ and low values of $\mathrm{Nb}, \mathrm{C}$.

In the same way, it is possible to use PCA to represent nodes of layer 2 as points of a space of dimensionality 14 (13 being this time the number of nodes of layer 1 plus one for the bias).

\subsection{Justification of Wrongly Modelled Cases}

After the training has been found optimum and stopped at a given number of iteration (for example at iteration 8000 for the ultimate strength $R$ ) residuals $\left(R_{\text {mes }}-R_{\text {est }}\right)$ are computed.

In a previous paper ${ }^{5)}$ we mentioned that the significant part of $\sigma$ values given at Table 1 , were due to a limited number of cases wrongly estimated by the NN approach and this situation was rather independent of the chosen structure.

On Fig. 6, 22 coils characterised by the ultimate strength residuals greater than $40 \mathrm{~N} / \mathrm{mm}^{2}$ after $\mathrm{NN}$ modelling have been visualised with a different symbol.

The examination of the different factorial plots $F_{12}$, $F_{13} \cdots$ suggests that most of those cases are in $p$-space unexplored areas during the training phase. This situation is illustrated in Fig. 6 for a set of four cases having high positive co-ordinates on axis $F_{1}$.

Another way to characterise the results is to calculate

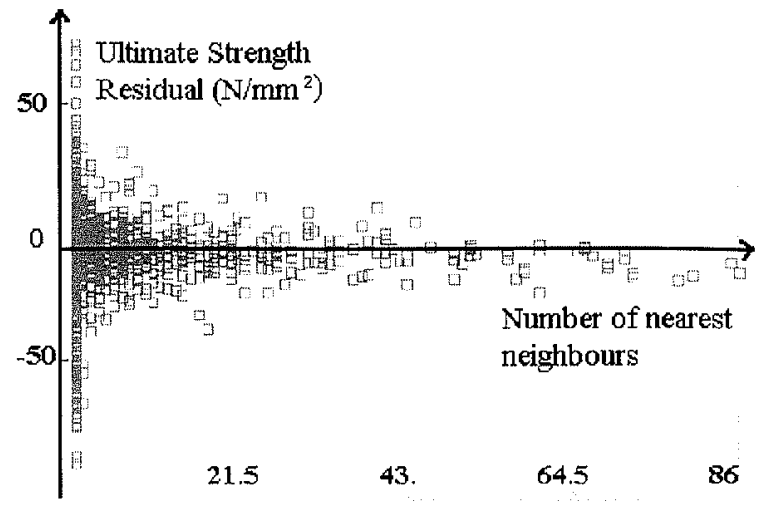

Fig. 9. Evolution of ultimate strength residuals with the number of nearest neighbours in $\mathrm{R}^{\mathrm{p}}$.

for each case of the validation database the number of nearest neighbours belonging to the learning database and situated in a fixed radius $p$-ball. Under the choice of an appropriate radius values, resulted into the graph of Fig. 9 suggesting that essential part of wrongly modelling cases have no or only very few neighbours in p-space.

It is also possible to calculate the distance of each case of the validation database to the nearest neighbour in the learning database $\left(d_{\mathrm{VL}}\right)$ : In so doing, we can observe that the standard error of estimate of ultimate strength $\left(\sigma_{R}\right)$ calculated for the validation data set decreases if the cases corresponding to $d_{\mathrm{VL}}$ greater than a fixed value are deleted.

For the two validation data sets (2197 patterns), if 68 cases corresponding to $d_{\mathrm{VL}}>0.15$ are deleted, the standards of estimate for $\mathrm{R}, \mathrm{Re}$ and $\mathrm{El}$ calculated on the 2129 remaining cases are respectively $12.5 \mathrm{~N} / \mathrm{mm}^{2}$, $20.2 \mathrm{~N} / \mathrm{mm}^{2}$ and $3.2 \%$. As a consequence, the quality results appear rather similar on validation and learning databases.

\section{Conclusions}

Neural Networks are increasingly considered as a powerful alternative tool with regard to statistical multivariate techniques in modelling steel characteristics. In this paper, Neural Networks were used to predict the mechanical properties of carbon and microalloyed steels produced at Cockerill-Sambre (Carlam) Steel Plant.

The determination of the model required to be assessed on the basis of independent data sets, was observed better than those obtained by linear multiple regression. However, the quality of results during the generalisation step can be explained by the size of the training data set and by the use of statistical data preparation realised before the analysis. In this way, Multivariate Data Analysis remains an essential step before, during and after NN modelling.

For a given set of variables selected for the modelling, neural networks properly applied can suggest a $\sigma$ limit value under which it is not possible to go down and which is largely explained by the dispersion measures of mechanical properties. 


\section{Acknowledgements}

The authors are grateful to Cockerill-Sambre Carlam Steel Plant for providing data and useful discussion.

\section{REFERENCES}

1) D. T. Gawne and G. M. Lewis: Mater. Technol., 183 (1985), 183.

2) C. Dumortier, P. Lehert, P. Donfut, J. M. Dullier and J. Marot: Rev. Métall.-CIT, Novembre (1992), 89, 1015.

3) D. J. Naylor: Ironmaking Steelmaking, 16 (1989), 246.

4) P. Lehert, C. Dumortier and P. Donfut: Ironmaking Steelmaking, 23 (1996), 25.

5) C. Dumortier, P. Lehert, P. Krupa and A. Charlier: Mater. Sci. Forum, Vols. Trans Tech Publications, Switzerland, (1998), 284, 393.

6) Stephen P. Curram and John Mingers: J. Opl. Res. Soc., 45 (1994), 440.
7) B. H. V. Topping, A. I. Khan and A. Bahreininejad: Comput. \& Struct., 63/4 (1997), 693.

8) S. T. Welstead: Neural Network and Fuzzy Logic Applications in C/C + +, John Wiley \& Sons, New York, (1994), 63.

9) D. E. Rumelhart and J. E. McClelland: Parallel Distributed Process, UHI, MIT Press, Cambridge, 1 (1986), 318.

10) Yhu-Jen Hwu, Yeong-Tsuen Pan and John G. Lenard: Steel Res., 67 (1996), 59.

11) P. F. Velleman and C. Hoaglin: Applications Basics, and Computing of Exploratory Data Analysis, Duxbury Press, Boston MA, (1981).

12) N. R. Draper and Smith: Applied Regression Analysis, John Wiley \& Sons, New York, (1969), 64.

13) H. Hartman: Modern Factor Analysis, 2nd Edition, University Press, Chicago, (1967).

14) J. Lefebvre: Introduction aux Analyses Statistiques Multidimensionnelles. Editions Masson, (1983), 228. 